\title{
The Full Scope of Family Physicians' Work Is Not Reflected by Current Procedural Terminology Codes
}

\author{
Richard A. Young, MD, Sandy Burge, PhD, Kaparaboyna Ashok Kumar, MD, FRCS, \\ and Jocelyn Wilson, MD
}

Background: The purpose of this study was to characterize the content of family physician (FP) clinic encounters, and to count the number of visits in which the FPs addressed issues not explicitly reportable by 99211 to 99215 and 99354 Current Procedural Terminology (CPT) codes with current reimbursement methods and based on examples provided in the CPT manual.

Methods: The data collection instrument was modeled on the National Ambulatory Medical Care Survey. Trained assistants directly observed every other FP-patient encounter and recorded every patient concern, issue addressed by the physician (including care barriers related to health care systems and social determinants), and treatment ordered in clinics affiliated with 10 residencies of the Residency Research Network of Texas. A visit was deemed to include physician work that was not explicitly reportable if the number or nature of issues addressed exceeded the definitions or examples for 99205/99215 or $99214+99354$ or a preventive service code, included the physician addressing health care system or social determinant issues, or included the care of a family member.

Results: In 982 physician-patient encounters, patients raised 517 different reasons for visit (total, 5278; mean, 5.4 per visit; range, 1 to 16) and the FPs addressed 509 different issues (total issues, 3587; mean, 3.7 per visit; range, 1 to 10). FPs managed 425 different medications, 18 supplements, and 11 devices. A mean of 3.9 chronic medications were continued per visit (range, 0 to 21) and 4.6 total medications were managed (range, 0 to 22). In 592 (60.3\%) of the visits the FPs did work that was not explicitly reportable with available CPT codes: 582 (59.3\%) addressed more numerous issues than explicitly reportable, 64 (6.5\%) addressed system barriers, and $13(1.3 \%)$ addressed concerns for other family members.

Conclusions and relevance: FPs perform cognitive work in a majority of their patient encounters that are not explicitly reportable, either by being higher than the CPT example number of diagnoses per code or the type of problems addressed, which has implications for the care of complex multi-morbid patients and the growth of the primary care workforce. To address these limitations, either the CPT codes and their associated rules should be updated to reflect the realities of family physicians' practices or new billing and coding approaches should be developed. (J Am Board Fam Med 2017;30:724-732.)

Keywords: Ambulatory Care, Current Procedural Terminology, Family Physicians, Health Care Financing, Insurance, Physician's Office, Practice Management, Physicians' Practice Patterns, Primary Health Care

Primary care is complex. ${ }^{1,2}$ Many variables influence family physicians' (FP) medical decision making beyond acute symptoms and chronic diseases

This article was externally peer reviewed.

Submitted 12 December 2016; revised 10 April 2017; accepted 27 April 2017.

From the Department of Family Medicine, JPS Health Network, Fort Worth, TX (RAY); Department of Family Medicine, University of Texas Health Science Center at San Antonio, San Antonio, TX (SB, KAK); Baylor Family Medicine Residency, Garland, TX (JW).

Funding: This project was funded with the help of the Texas Academy of Family Physicians Foundation. The project was also supported by the National Center for Advancing including patients' socioeconomic status, sex, culture/ethnicity, environment/ecology, health behaviors, functional status, cognitive impairment, men-

\footnotetext{
Translational Sciences, National Institutes of Health (NIH), through Grant UL1 TR001120. The content is solely the responsibility of the authors and does not necessarily represent the official views of the NIH.

Conflict of interest: RAY is the sole owner of Sentire, LLC, which is developing a novel method for family physicians to document, code, and bill for their work.

Corresponding author: Richard A. Young, MD, Department of Family Medicine, JPS Health Network, $1500 \mathrm{~S}$. Main, Fort Worth, TX 76104 (E-mail: Ryoung01@ jpshealth.org).
} 
tal disorders, addiction, social capital, family relationships, personal priorities, and health systems barriers. ${ }^{3-9}$

The American Medical Association's (AMA) book, Current Procedural Terminology (CPT), is the only system recognized by the Center for Medicare and Medicaid Services (CMS) and is used by most insurance companies for physicians to code their bills for third-party reimbursement. ${ }^{10}$ In 1995 and 1997, CMS published guidelines for documentation, coding, and billing for evaluation and management (E\&M) services provided to its beneficiaries that are still used today. ${ }^{11,12}$

CMS states in its E\&M rules that “... to receive payment from Medicare for a service, the service must... be considered reasonable and necessary,"11 but there is little further explanation of what exactly defines "necessary." This responsibility has largely been given to the 8 Medicare Administrative Contractors (MACs). To define medical necessity, the Web site of each MAC refers to the CMS guidelines, the CPT codes, and some specifically mention Appendix $\mathrm{C}$ of the CPT manual that includes numerous clinical examples for each $\mathrm{E} \& \mathrm{M}$ code.

The most common CPT codes used by FPs are 99213 and $99214 .{ }^{13}$ Under the 1997 CMS E\&M rules and CPT Appendix C examples, a 99213 can be billed if a physician sees a patient for 1 stable chronic disease (eg, "stable cirrhosis of the liver"). ${ }^{10}$ A 99214 can be billed if a FP cares for a patient with 1 chronic disease not in optimal control (eg, "stable angina, 2 months post myocardial infarction, who is not tolerating 1 of his medications"). ${ }^{10}$ There are no examples in the CMS E\&M guidelines or CPT book of how to code for a patient with 3 chronic conditions in poor control, for example, or a patient with 3 chronic diseases and 3 new symptoms, for another example.

This issue is important because 1) previous observational studies of FP's found that they actually manage a mean of 2.5 to 3.1 diagnoses $^{14-16}, 3.9$ to 6 for elderly patients and 4.6 for patients with diabetes per clinic visit ${ }^{15-17}$;2) some insurance forms only permit 4 diagnoses lines for any single CPT charge code; and 3) CPT codes are directly tied to relative value units (RVUs), which are commonly used by practice groups to guide compensation. ${ }^{18}$ Therefore, work that is over and beyond that found in the CPT examples, and thus not explicitly reportable using current CPT Codes with its E\&M rules, is neither captured by billing data nor paid under an RVU-based productivity model.

The purpose of our study was to thoroughly describe the issues addressed in FP-patient office visits and to determine the number of these visits in which the physicians provided cognitive work that was not explicitly reportable under CPT (based on the E\&M rules and connected examples in the CPT Coding Manual).

\section{Methods}

\section{Study Population}

This was a cross-sectional observational study of primary care visits in clinics of 10 family medicine residencies that are members of the Residency Research Network of Texas (RRNeT).

Patient-physician encounters were purposefully sampled at the level of the observed physician. A true randomization of physicians was believed to be impractical given the nature of the subject physicians' erratic clinic schedules. However, local site RRNeT members and their associated research assistants were asked to observe as wide a variety of physicians as possible and to preferentially observe FP faculty whenever possible, followed by third- or second-year residents. Students shadowed each selected physician for a half-day clinic session and recorded every second patient visit. Data collection occurred in May and June 2015 toward the end of the academic year, which meant that observed residents had 2 and 3 full years of clinical experience.

\section{Observer Training and Measurements}

Visit observers/data collectors ("observers") were medical students enrolled at the University of Texas Health Science Center at San Antonio and Texas Tech Health Sciences University in Lubbock, Texas, plus 1 premed undergraduate student from a college in Washington state. Each volunteered to participate in a 4-week research elective during a normal break in their class schedule. Investigators scheduled a 1-day orientation and training session at the medical school in San Antonio, Texas to teach enrollment and consent procedures and review all study instruments. Before the training, 1 investigator filmed physician-patient interactions (both complex and simple visits) and used these during training so the observers could practice recording pertinent data. The investigator- 
trainers provided feedback to insure consistent data collection approaches among all observers.

This study used a visit survey to describe each patient's visit in multiple domains. The primary instrument was based on the National Ambulatory Medical Care Survey (NAMCS), which includes the reason for visit; new or continuing/follow-up patient, patient demographics, diagnoses, screening services, exams, medicines, procedures, nonmedical treatments, and referral patterns. ${ }^{19}$ Unlike NAMCS, there were no numeric limits on any of the categories in our study.

Observers were instructed to record all concerns brought up in the visit by either the patient or physician. These could include symptoms, diagnoses, socioeconomic concerns, health care system issues, or even concerns about people other than the patient (for example, a question about a child's health during an adult's scheduled visit). These concerns were recorded in a section titled Reasons for Visit (RFVs), which mirrored the NAMCS instrument. Observers were also asked to record all the concerns that were actually addressed by the physician during the visit (issues addressed), which also mirrors the NAMCS instrument. The investigators-trainers provided training on how to make this distinction during the session. For example, a patient may have mentioned a recent headache, but the physician spent no observed time taking a history of the headache or prescribing a medication for the headache. In this scenario, the headache would be recorded in the RFV section, but not the Issues Addressed (IAs) section. Further details on how a variety of these issues were classified are in the online Appendix.

\section{Observations}

Observers recorded data from every other encounter to allow time to complete all the study instruments for the index patient encounter and to decrease the burden of their presence on the observed physician. Observers were instructed to be a "fly on the wall" and to impact the encounter as little as possible. They were instructed to move to the corner of the examination room that was the least visible to the patient. A crucial component of data collection was the time required for the physician to complete the encounter, both face-to-face time and nonface time. Observers were instructed to not make any statements or ask the physician any questions until he or she was finished with that encounter and was ready to see the next patient. Although the physician was seeing the nonobserved patient, the observer would finish recording relevant data on article copies of the instruments and await the next eligible patient. The observer would explain the nature of the study and seek verbal consent from the patient and furnish a 1-page informational letter (signed consent was not required). If the patient declined to be observed, the next eligible patient was approached. At the end of each half-day clinic session, the observer transferred the written data onto a SurveyMonkey version of the instrument.

\section{Data Coding}

Free text data for RFVs, IAs, and medications was converted to numeric codes. Two of the investigators (RAY and SB) coded 1 of the categories and those codes were vetted by the other investigator. The RFV text was converted to a 5-digit code using the NAMCS nomenclature. IAs were converted to mostly International Classification of Disease version 9 (ICD-9) codes and recorded to 1 decimal place. If an IA did not have a relevant code, the International Classification of Primary Care scheme was used, or in a few cases, the investigators created study-specific codes. In the process of vetting the codes, RAY edited some of the observer entries to insure consistency of recording styles across the entire study. Less than $5 \%$ of data cells were altered and most involved eliminating duplicate entries or placing an element of data into the correct section.

Medications were coded using the Centers for Disease Control and Prevention New Ambulatory Care Drug Database System. ${ }^{20}$ Combinations of commonly available individual medications were counted as 2 separate medications. For example, a lisinopril/hydrochlorothiazide pill was counted as 2 medications. Combinations not commonly available as separate medications were counted as 1 medication, for example, codeine with acetaminophen or butalbital with caffeine and acetaminophen.

\section{CPT Reportable Classifications}

A full description of the determination of the appropriate CPT codes and whether one was considered explicitly reportable is in the online Appendix. Briefly, the number of acute and chronic issues addressed in the visit were compared with the descriptions of medical necessity clinical examples in 
the Appendix C of the CPT Manual for the codes 99211 to 99215 and 99201 to 99205 , which was also consistent with the CMS E\&M guideline that uses a point counting system over multiple tables. ${ }^{12}$ The extended care code 99354 was applied if the observed face time was greater than 55 minutes per CMS guidelines (25 minutes for a 99214 plus at least 30 minutes toward the 99354 charge) ${ }^{21}$ Complex chronic care coordination codes such as 99487 were not deemed usable because the CPT manual states, "... clinical staff time [may not be counted] ... on a day when the physician ... reports an E/M service ...."10 In addition, CMS and insurance companies do not pay for more than 1 CPT E\&M code submitted for services provided on the same day. Therefore, issues that were addressed beyond those covered by a 99214 or 99215 code plus either a preventive service code (eg, 99395) or a prolonged service code (99354) were classified as not explicitly reportable.

Other important aspects of a FP's work required to make complex medical decisions are also not explicitly included in the CPT coding criteria either by the CPT manual or CMS E\&M rules. Particularly, the time and expertise required to navigate health-care-system barriers and issues, and the expertise to manage patient concerns related to social determinants of health are not mentioned in either the CPT coding manual or the CMS E\&M rules. In addition, patient questions about the health of other family members not physically present are not reportable. Therefore, patient visits with 1 of these 3 issues were also counted as not explicitly reportable.

Preventive services such as a well woman visit were counted as being reportable using the appropriate preventive codes. This analysis did not include issues related to CMS rules of documentation elements (eg, bullet points of physical examination elements) also required to justify the various codes. Observers did not collect data on what the physicians actually documented.

\section{Data Analysis}

Results were analyzed using descriptive statistics. Group comparisons of continuous variables were assessed using independent samples $t$-tests or ANOVA as appropriate, and comparisons of all categorical data were analyzed using $\chi^{2}$. SPSS (Statistical Package for the Social Scientist) version 20 was used for analysis (SPSS Inc., Chicago, IL). All tests were 2 tailed and $\alpha$ levels were set at 0.05 to determine statistical significance.

This study was approved by the Institutional Review Board (IRB) of the University of Texas Health Science Center at San Antonio, Texas, and the individual residencies' IRBs where required.

\section{Results}

Nine-hundred eighty-two physician-patient ambulatory visits were observed and recorded. Patient and visit characteristics are shown (Table 1). The patients were majority White/Hispanic (54.3\%), female $(61.4 \%)$, with a mean age of 46.4 years, ranging from newborns to elders age 90 years and above.

Patients brought 517 different reasons for the visit (5278 total RFVs; mean, 5.4 per visit; range, 1 to 16$)$ and the physicians addressed 509 different issues/diagnoses (3587 total issues; mean, 3.7 per visit; range, 1 to 10). Physicians managed 425 different medications, 18 supplements, and 11 devices. A mean of 3.9 chronic medications were continued per visit (range, 0 to 21), 0.7 new medications were prescribed (range, 0 to 11), 0.2 immunizations were administered (range, 0 to 6 ), and 4.6 total medications and immunizations were managed (range, 0 to 21). The most common reasons for visit and issues addressed are shown (Tables 2 and 3).

In $592(60.3 \%)$ of the visits, the physicians did work that was not explicitly reportable. In 582 of the visits $(59.3 \%)$, the physician addressed enough diagnoses to justify a 99214- or 99215-level code, plus additional issues in that visit that were not separately reportable, primarily traditional diagnoses. In 64 visits $(6.5 \%)$, the physicians addressed system barriers that are not reportable with CPT codes. Examples included calling an insurance company to see if a medication was covered and calling a referral clinic and/or physician directly after the standard referral process was unsuccessful in a previous visit. In 14 of the visits (1.4\%), physicians addressed social determinant barriers. An example was discussing medication options if the patient told the physician that the preferred medication was unaffordable. In 13 of the visits (1.3\%), the physicians addressed health concerns of other family members not present. For example, the visit was for the mother, but the physician also answered questions about a child's medications. A clinic visit could have more than 1 reason that issues were ad- 
Table 1. Patient and Visit Characteristics

\begin{tabular}{|c|c|}
\hline Characteristic & Results $(\mathrm{n}=982)$ \\
\hline Age, mean (SD) & $46.4(22.5)$ \\
\hline Male sex, No. (\%) & $380(38.6)$ \\
\hline \multicolumn{2}{|l|}{ Race/Ethnicity, No. (\%) } \\
\hline White/Hispanic & $532(54.3)$ \\
\hline White/Non-Hispanic & $233(23.7)$ \\
\hline Black & $165(16.8)$ \\
\hline Asian & $37(3.8)$ \\
\hline Hawaiian/Pacific Islander & $6(0.6)$ \\
\hline American Indian & $7(0.7)$ \\
\hline Body mass index, mean (SD) & $31.3(8.0)$ \\
\hline \multicolumn{2}{|l|}{ Blood pressure, mean (SD) } \\
\hline Systolic & $126.5(19.8)$ \\
\hline Diastolic & $73.6(11.4)$ \\
\hline \multicolumn{2}{|l|}{ History of disease, No. (\%) } \\
\hline Hypertension & $400(40.7)$ \\
\hline Obesity & $306(31.2)$ \\
\hline Diabetes & $277(28.2)$ \\
\hline Hyperlipidemia & $272(27.7)$ \\
\hline Depression & $178(18.1)$ \\
\hline Arthritis & $138(14.1)$ \\
\hline Anxiety & $109(11.1)$ \\
\hline Headache & $93(9.5)$ \\
\hline Asthma & $92(9.5)$ \\
\hline COPD & $56(5.7)$ \\
\hline Coronary artery disease & $50(5.1)$ \\
\hline Chronic kidney disease & $49(5.0)$ \\
\hline \multicolumn{2}{|l|}{ PCP relationship, No. (\%) } \\
\hline Patient saw personal physician & $534(54.4)$ \\
\hline $\begin{array}{l}\text { Established patient, but did not see } \\
\text { personal physician }\end{array}$ & $333(33.9)$ \\
\hline $\begin{array}{l}\text { Neither the FP nor the practice was } \\
\text { the patient's primary care physician } \\
\text { prior to the observed visit }\end{array}$ & $52(5.3)$ \\
\hline $\begin{array}{l}\text { Unknown prior practice or FP-patient } \\
\text { relationship }\end{array}$ & $62(6.3)$ \\
\hline \multicolumn{2}{|l|}{ Practitioner type, No. (\%) } \\
\hline Faculty physician & $313(31.9)$ \\
\hline PGY4/fellow & $18(1.8)$ \\
\hline PGY3 & $372(37.9)$ \\
\hline PGY2 & $262(26.7)$ \\
\hline PGY1 & $8(0.8)$ \\
\hline $\begin{array}{l}\text { Electronic medical record visit, No. } \\
\quad(\%)\end{array}$ & $978(99.6)$ \\
\hline Number of reasons for visit & $5.4(2.8)$ \\
\hline $\begin{array}{l}\text { Number of issues addressed by the } \\
\text { physician }\end{array}$ & $3.7(2.1)$ \\
\hline
\end{tabular}

COPD, chronic obstructive pulmonary disease; FP, family physician; PCP, primary care physician; PGY, post graduate year; $\mathrm{SD}$, Standard Deviation.
Table 2. Most Common Reasons for Visit

\begin{tabular}{lcc}
\hline Reason for Visit & $\begin{array}{c}\text { Visits } \\
(\%)\end{array}$ & $\begin{array}{c}\text { RFVs } \\
(\%)\end{array}$ \\
\hline Hypertension & 19.4 & 3.6 \\
Diabetes mellitus & 14.8 & 2.8 \\
Problem with access to medical care & 11.8 & 2.2 \\
Findings of blood tests (eg, cholesterol) & 11.7 & 2.2 \\
Anxiety & 8.8 & 1.6 \\
Headache & 8.4 & 1.5 \\
Back pain, ache, soreness, discomfort & 8.4 & 1.5 \\
Medical counseling, NOS & 8.4 & 1.5 \\
Cough & 7.7 & 1.4 \\
Administrative issues, paperwork & 7.0 & 1.3 \\
Depression & 6.8 & 1.3 \\
Patient seeks referral to a specialist & 6.6 & 1.2 \\
Vertigo, dizziness & 6.3 & 1.2 \\
Diet and nutritional counseling, also & 6.3 & 1.2 \\
$\quad$ exercise and weight-loss counseling & & \\
Shortness of breath & 6.0 & 1.1 \\
General medical exam & 5.8 & 1.1 \\
Insomnia & 5.5 & 1.0 \\
For results of blood glucose tests & 5.4 & 1.0 \\
For results of cholesterol and triglycerides & 5.4 & 1.0 \\
Heartburn and indigestion (GERD, & 5.2 & 1.0 \\
$\quad$ dyspepsia) & & \\
Tiredness, exhaustion & 5.1 & 0.9 \\
Constipation & 5.0 & 0.9 \\
For radiological findings & 5.0 & 0.9 \\
Well baby examination & 4.8 & 0.9 \\
\hline
\end{tabular}

GERD, gastroesophageal reflux disease; NOS, no otherwise specified; RFV, reason for visit.

dressed that were not explicitly reportable. Other patient and physician characteristics and their association with visit reportability are shown (Table 4).

There were significant differences in the patient populations by multiple measures between the 10 clinic sites $(P<.001$ for many comparisons). Some clinics had older patients with more chronic diseases and addressed more issues per visit. A table comparing key patient characteristics and outcomes by clinic site is shown in the online Appendix.

The adequacy of reportability of diagnoses and issues did not differ by physician training level, physician clinical experience, established patient status, patient sex, or patient race/ethnicity. Details of these findings are listed in Table 4. The adequacy of the reportability was significantly associated with the clinic site, whether the physician was the patient's primary physician, the patient's previous number of visits in the last year, the patient's age, and the patient's body mass index. 
Table 3. Most Common Issues Addressed by the Family Physician

\begin{tabular}{lcc}
\hline & $\begin{array}{c}\text { Visits } \\
(\%)\end{array}$ & $\begin{array}{l}\text { IAs } \\
\text { Issues Addressed }\end{array}$ \\
\hline Essential hypertension & 21.8 & 6.0 \\
Diabetes mellitus & 21.2 & 5.8 \\
Encounters for administrative purposes & 15.7 & 4.3 \\
Disorders of lipid metabolism & 9.1 & 2.5 \\
Anxiety, dissociative, and somatoform & 6.7 & 1.8 \\
$\quad$ disorders & & \\
Allergic rhinitis & 6.6 & 1.8 \\
Osteoarthrosis and allied disorders & 6.6 & 1.8 \\
Follow-up examination & 6.4 & 1.8 \\
Depressive disorder & 6.3 & 1.7 \\
Health supervision of infant or child & 5.5 & 1.5 \\
Other and unspecified disorders of the back & 5.1 & 1.4 \\
Symptoms involving respiratory system and & 5.1 & 1.4 \\
$\quad$ other chest symptoms & & \\
Problems related to lifestyle & 5.0 & 1.4 \\
Diseases of esophagus & 4.9 & 1.3 \\
Acquired hypothyroidism & 4.6 & 1.3 \\
Other and unspecified disorders of joint & 4.4 & 1.2 \\
General medical examination (usually well & 4.1 & 1.1 \\
$\quad$ woman) & & \\
Obesity & 3.9 & 1.1 \\
Other symptoms involving abdomen & 3.6 & 1.0 \\
Nonspecific findings on examination of & 3.6 & 1.0 \\
$\quad$ blood & 3.6 & 1.0 \\
Normal pregnancy & 3.3 & 0.9 \\
Other disorders of soft tissues & 3.3 & 0.9 \\
Other symptoms involving nervous and & & \\
$\quad$ musculoskeletal systems & 3.3 & 0.9 \\
Special investigations and exams & 0.9 \\
Other disorders of urethra and urinary tract & & \\
Symptoms concerning nutrition metabolism & 3.2 & \\
$\quad$ and development & & \\
\hline & & \\
\hline
\end{tabular}

IA, issue addressed.

\section{Conclusions}

In this study of $982 \mathrm{FP}$-patient ambulatory visits, patients raised 517 different reasons for the visit, the FPs addressed 508 different issues, and the FPs managed 425 different medications. In $60.3 \%$ of the visits the primary care physicians (PCPs) did work that was not explicitly reportable, the majority occurring because the physician addressed a greater number of diagnoses than are explicitly reportable using the CPT codes, followed by visits where the physician addressed system barriers.

Our findings are consistent with a 2011 article using NAMCS data comparing the complexity of primary care with cardiology and psychiatry, where 491 different diagnoses were identified in primary care. $^{22}$ Our study also found that the number of issues addressed per visit was similar to the previous studies looking at higher risk elderly and diabetic populations. ${ }^{15,17}$

Potential CPT codes 99205 and 99215 were counted even though they are rarely reported in primary care ${ }^{13}$, and when these codes are submitted, the majority are denied. ${ }^{23}$ Properly applying 99205/99215 codes are also problematic because under the management option column in the CPT guidelines. It does not define what an "extensive" number of diagnoses or management options means. Also, the examples in the CPT coding manual for a 99215 requires exacerbations of medical illness so severe that patients often require hospitalization or major intervention, which would be unusual in a family medicine office ${ }^{24}$, and applies regardless of the social complexity or number of diagnoses handled. Of note, CMS does not pay the primary physician for both the outpatient and inpatient $\mathrm{E} \& \mathrm{M}$ codes on the same day. ${ }^{12}$

Our study adds to NAMCS format used in previous studies. NAMCS originally allowed only 3 reasons for visit, 3 diagnoses, and 7 medications. The 2014 version of the NAMCS survey addresses some of these shortcomings (http://www.cdc.gov/nchs/ ahcd/ahcd_questionnaires.htm). The number of reasons for visit and diagnoses has expanded to 5 , and the list for medications can now accommodate 30 medicines. The lists of exams, lab and imaging tests, procedures, and treatments have expanded as well. Our study suggests that NAMCS should expand the reasons for visit and number of diagnoses further.

The hours FPs work per week is near the median of all physicians ${ }^{25}$, but their average personal income is approximately one third that of the highest-paid physicians and is a little more than half the average of nonprimary-care physicians. ${ }^{26}$ An analysis of physician incomes concluded that the Medicare fee schedules that arise from CMS's E\&M rules directly explain this income discrepancy, not inflated fees to nonprimary care physicians in the private market. ${ }^{27}$ Our findings show that even before the CMS E\&M rules are considered, the AMA's CPT codes give the FP no mechanism to fully describe and report the work performed in the majority of clinic visits. To the authors' knowledge, no other developed country uses the CPT system for primary care physicians to report their work.

We did not attempt to account for physician work not directly involved with the observed clinic 
Table 4. Characteristics of Visits with at Least One Diagnosis or Issue That Was Not Explicitly Reportable

\begin{tabular}{|c|c|c|c|}
\hline \multicolumn{2}{|l|}{ Factor } & $\begin{array}{l}\text { with Element Not Explicitly } \\
\text { Reportable, No. (\%) }\end{array}$ & $P$ Value \\
\hline \multicolumn{4}{|l|}{ FP status } \\
\hline \multicolumn{2}{|l|}{ Patient saw personal physician } & $333(62.4)$ & \\
\hline \multicolumn{2}{|c|}{ Established patient, but did not see personal physician } & $204(61.3)$ & .025 \\
\hline \multicolumn{2}{|c|}{$\begin{array}{l}\text { Neither the FP nor the practice was the patient's primary } \\
\text { care physician prior to the observed visit }\end{array}$} & $26(50.0)$ & \\
\hline \multicolumn{2}{|c|}{ Unknown prior practice or FP-patient relationship } & $28(45.2)$ & \\
\hline \multicolumn{4}{|c|}{ Continuity status } \\
\hline \multicolumn{2}{|l|}{ Established patient } & $533(60.4)$ & .83 \\
\hline \multicolumn{2}{|l|}{ New patient } & $59(59.0)$ & \\
\hline \multicolumn{4}{|l|}{ Practitioner type, No. (\%) } \\
\hline \multicolumn{2}{|l|}{ Faculty physician } & $201(64.2)$ & \\
\hline \multicolumn{2}{|l|}{ PGY4/fellow } & $11(61.1)$ & \\
\hline \multicolumn{2}{|l|}{ PGY3 } & $216(58.1)$ & .53 \\
\hline \multicolumn{2}{|l|}{ PGY2 } & $153(58.4)$ & \\
\hline \multicolumn{2}{|l|}{ PGY1 } & $5(62.5)$ & \\
\hline \multicolumn{4}{|l|}{ Patient gender, No. (\%) } \\
\hline \multicolumn{2}{|l|}{ Male } & $225(59.4)$ & .66 \\
\hline \multicolumn{2}{|l|}{ Female } & $366(60.8)$ & \\
\hline \multicolumn{4}{|l|}{ Patient race/ethnic group } \\
\hline \multicolumn{2}{|l|}{ White/Hispanic } & $321(60.3)$ & \\
\hline \multicolumn{2}{|l|}{ White/non-Hispanic } & $131(56.2)$ & \\
\hline \multicolumn{2}{|l|}{ Black } & $113(68.5)$ & .056 \\
\hline \multicolumn{2}{|l|}{ Asian } & $17(45.9)$ & \\
\hline \multicolumn{2}{|l|}{ Hawaiian/Pacific Islander } & $5(83.3)$ & \\
\hline \multicolumn{2}{|l|}{ American Indian } & $4(57.1)$ & \\
\hline \multicolumn{4}{|l|}{ Age (years) } \\
\hline \multicolumn{2}{|l|}{$<65$} & $445(58.1)$ & .005 \\
\hline \multicolumn{2}{|l|}{$\geq 65$} & $144(68.9)$ & \\
\hline Factor & $\begin{array}{l}\text { Mean if Visit } \\
\text { Reportable (SD) }\end{array}$ & $\begin{array}{c}\text { Mean if Visit Not Explicitly } \\
\text { Reportable (SD) }\end{array}$ & $P$ Value \\
\hline $\begin{array}{l}\text { Number of visits to that clinic in the } \\
\text { previous } 12 \text { months }\end{array}$ & $3.7(4.1)$ & $4.3(4.2)$ & .051 \\
\hline Patient age (years) & $39.3(24.6)$ & $51.0(19.7)$ & $<.001$ \\
\hline Patient body mass index & $30.4(6.9)$ & $31.7(8.5)$ & .020 \\
\hline Clinical experience of the physician (years) & $7.2(9.0)$ & $7.0(8.4)$ & .81 \\
\hline
\end{tabular}

FP, family physician; PGY, post graduate year; SD, Standard Deviation.

visits, such as fielding telephone calls, emails, prescription refills, and reviewing lab reports, imaging reports, and consultant reports. ${ }^{28}$ Previous studies estimated that this work consumed between $20 \%$ and $50 \%$ of a FP's time that is not compensated and often has no CPT code to describe the work. ${ }^{29,30}$

Our results help explain previous research on the problems identified by FPs in the current CMS E\&M rules and CPT codes. ${ }^{31}$ Participants believed they were not adequately paid for taking care of patients with complex needs, which could arise from factors including multiple chronic diseases, difficult patients, family/social factors, language/ cultural barriers, and financial barriers. They believed a better coding system should take into account the time required to provide care for complex patients more than the CMS E\&M system allows. ${ }^{32}$ Our findings help enumerate how often this happens in an average clinic day.

\section{Limitations}

Our study was limited by its observational nature. Observers were mostly consistent in their classification of observed visits, but visits were not audio re- 
corded to cross check the observations as other studies have done. ${ }^{14}$ In addition, 1 day of training may not have been adequate to achieve maximal fidelity of observations and recordings. Patient and provider behaviors may have been altered with the presence of a third person in the examination room: the Hawthorne effect. Our study has the strength of recording more reasons for visits and issues addressed by a neutral observer and not relying on electronic medical record (EMR) or billing records, which have been shown in previous studies to undercount the number of issues addressed. ${ }^{15}$

We tried to err on the side of undercounting issues addressed. For example, well child, well woman, and prenatal care visits were counted as 1 issue, although there are many subparts to these visits. The unpredictable nature of the day of a FP precludes precise definitions of workflow. ${ }^{33,34} \mathrm{Be}-$ cause the researchers discussed the importance with the observers of capturing all the issues that were mentioned in a clinic visit, it is possible the observers overdescribed some of the encounters. However, investigators inspected observers' text descriptions of reasons for visit, issues addressed, and medications then cross checked these descriptions to reduce redundancies or irrelevancies, and applied appropriate descriptive codes.

The analysis of comparing the issues addressed in the clinic visit and determining whether those issues were fully reportable with an existing CPT code was made difficult by the vague and sometimes conflicting guidance and examples listed in the CPT manual, which may lead to interobserver variability bias. We attempted to minimize this by completing several rounds of data vetting between 2 of the investigators (RAY and SB).

\section{Implications}

Because the highest-cost Medicare and Medicaid patients are those with multiple chronic diseases ${ }^{35}$, creating a payment system that encourages thorough primary care for these patients would be desirable, which our study suggests is not the current situation. Our findings also show the limitations of using our current system to account for the work performed by FPs. This concern is in addition to the observation that RVUs (based on CPT coding with examples) preferentially reward procedural work over cognitive work. ${ }^{36,37}$
The researchers thank our medical student research observers: Clayton Bishop, Caroline Stephens, Tanner Campbell, Nicholas Inman, Jade Law, Brenda Chavez, Daniela Ortiz, Katie Molina, Tiffany Brown, Shannon Brougher, and Thomas Heydon. We also thank our administrative assistants, Colleen Dolan and Jennifer Daniels.

To see this article online, please go to: http://jabfm.org/content/ 30/6/724.full.

\section{References}

1. Katerndahl D, Parchman M, Wood R. Perceived complexity of care, perceived autonomy, and career satisfaction among primary care physicians. J Am Board Fam Med 2009;22:24-33.

2. Katerndahl D, Parchman M, Wood R. Trends in the perceived complexity of primary health care: A secondary analysis. J Eval Clin Pract 2010;16:1002-1008.

3. Grant RW, Ashburner JM, Hong CS, et al. Defining patient complexity from the primary care physician's perspective: A cohort study. Ann Intern Med 2011; 155:797-804.

4. Nardi R, Scanelli G, Corrao S, Iori I, Mathieu G, Cataldi Amatrian R. Co-morbidity does not reflect complexity in internal medicine patients. Eur J Intern Med 2007;18:359-368.

5. Peek CJ, Baird MA. Point-of-care complexity assessment helps primary care clinicians identify barriers to improved health and craft integrated care plans. Rockville, MD: Agency for Healthcare Research and Quality; 2009.

6. Safford MM, Allison JJ, Kiefe CI. Patient complexity: More than comorbidity. The vector model of complexity. J Gen Intern Medic 2007;22(Suppl 3): 382-390.

7. Schaink AK, Kuluski K, Lyons RF, et al. A scoping review and thematic classification of patient complexity: Offering a unifying framework. J Comorbidity 2012;2:1-9.

8. Shippee ND, Shah ND, May CR, Mair FS, Montori VM. Cumulative complexity: A functional, patientcentered model of patient complexity can improve research and practice. J Clin Epidemiol 2012;65: 1041-1051.

9. Smucker DR, Zink T, Susman JL, Crabtree BF. A framework for understanding visits by frequent attenders in family practice. J Fam Pract 2001;50:847852.

10. American Medical Association. CPT 2014 Standard Edition (Current Procedural Terminology). Chicago, IL: American Medical Association; 2014.

11. Centers for Medicare and Medicaid Services. 1997 Documentation Guidelines for Evaluation and Management Services. Available from: http://www.cms. hhs.gov/MLNProducts/Downloads/MASTER1. pdf.

12. Centers for Medicare \& Medicaid Services. Evaluation and Management Services Guide. Available 
from: https://www.cms.gov/Outreach-and-Education/ Medicare-Learning-Network-MLN/MLNProducts/ Downloads/eval-mgmt-serv-guide-ICN006764.pdf. Accessed April 26, 2016.

13. State of Vermont Department of Banking I, Securities, and Health Care Administration. 2009 Provider reimbursement report: Primary care services. Available from: http://www.leg.state.vt.us/reports/2010 ExternalReports/251708.pdf. Accessed March 3, 2016.

14. Stange KC, Zyzanski SJ, Jaén CR, et al. Illuminating the 'black box'. A description of 4454 patient visits to 138 family physicians. J Fam Pract 1998;46:377-389.

15. Beasley JW, Hankey TH, Erickson R, et al. How many problems do family physicians manage at each encounter? A WReN study. Ann Fam Med 2004;2: 405-410.

16. Flocke SA, Frank SH, Wenger DA. Addressing multiple problems in the family practice office visit. J Fam Pract 2001;50:211-216.

17. Tai-Seale M, McGuire TG, Zhang W. Time allocation in primary care office visits. Health Serv Res 2007;42:1871-1894.

18. Kaplan KC. Physician compensation fair market value: The perils of "compensation per work RVU" benchmarks. Healthcare Financial Management Association. Available from: http://www.veralon.com/ articles/physician-compensation-fair-market-valuethe-perils/. Accessed Oct 9, 2017.

19. Centers for Disease Control and Prevention. Questionnaires, datasets, and related documentation. Available from: http://www.cdc.gov/nchs/ahcd/ ahcd_questionnaires.htm. Accessed Feb 25, 2016.

20. Centers for Disease Control and Prevention. New ambulatory care drug database system. Available from: http://www2.cdc.gov/drugs/applicationnav1.asp. Accessed Feb 25, 2016.

21. MLN Matters. Center for Medicare \& Medicaid Services. Prolonged Services (Codes 99354-99359). Available from: https://www.cms.gov/Outreachand-Education/Medicare-Learning-Network-MLN/ MLNMattersArticles/downloads/mm5972.pdf. Accessed April 5, 2017.

22. Katerndahl D, Wood R, Jaén CR. Family medicine outpatient encounters are more complex than those of cardiology and psychiatry. J Am Board Fam Med 2011;24:6-15

23. National Government Services. NGS Focused Audit Review for CPT 99215, April-June 2015. Available from: https://www.ngsmedicare.com/ngs/portal/ ngsmedicare/newngs/home-lob/pages/compliance andaudits/medical-review/medical-review-focusareas/medical-review-focus-areas-detail/. Accessed March 23, 2016.
24. Edsall RL, Moore KJ. Thinking on paper: Documenting decision making. Fam Pract Manag 2010; 17:10-15.

25. Leigh JP, Tancredi D, Jerant A, Kravitz RL. Annual work hours across physician specialties. Arch Intern Med 2011;171:1211-1213.

26. Medical Group Management Association. MGMA 2015 Physician Compensation and Production. Available from: http://www.mgma.com/Libraries/Assets/ Industry\%20Data/Survey\%20Reports/MGMAPhysician-Compensation-Executive-Summary-Report. pdf?ext=.pdf. Accessed Oct 9, 2017.

27. Berenson RA, Zuckerman S, Stockley K. What if All physician services were paid under the medicare fee schedule? March 2010. Available from: www.urban. org/UploadedPDF/412051_physcian_service.pdf. Accessed Oct 9, 2012.

28. Baron RJ. What's keeping us so busy in primary care? A snapshot from one practice. N Engl J Med 2010;362:1632-1636.

29. Chen MA, Hollenberg JP, Michelen W, Peterson JC, Casalino LP. Patient care outside of office visits: A primary care physician time study. J Gen Intern Med 2011;26:58-63.

30. Gottschalk A, Flocke SA. Time spent in face-to-face patient care and work outside the examination room. Ann Fam Med 2005;3:488-493.

31. Young RA, Bayles B, Hill JH, Kumar KA. Family physicians' opinions on the primary care documentation, coding, and billing system: A qualitative study from the Residency Research Network of Texas. Fam Med 2014;46:378-384.

32. Young RA, Bayles B, Hill JH, Kumar KA, Burge S. Family physicians' suggestions to improve the documentation, coding, and billing system: A study from the Residency Research Network of Texas. Fam Med 2014;46:470-472.

33. Holman GT, Beasley JW, Karsh BT, Stone JA, Smith PD, Wetterneck TB. The myth of standardized workflow in primary care. J Am Med Inform Assoc 2016;23:29-37.

34. Linzer M, Manwell LB, Williams ES, et al. Working conditions in primary care: Physician reactions and care quality. Ann Intern Med 2009;151:28-36, W26-29.

35. Anderson G. Chronic conditions: Making the case for ongoing care. Available from: https://www.rwjf. org/en/library/research/2010/01/chronic-care. html. Accessed Oct 9, 2017.

36. Sinsky CA, Dugdale DC. Medicare payment for cognitive vs procedural care: minding the gap. JAMA Intern Med 2013;173:1733-1737.

37. Berenson, RA, Goodson JD. Finding value in unexpected places-fixing the medicare physician fee schedule. N Engl J Med 2016;374:1306-1309. 


\section{Appendix \\ Reasons for visit and Issues Addressed classifi- cation and coding scheme}

\section{Reasons for visit (RFVs)}

RFVs were recorded as patient-oriented as possible: i.e., in their own words. If a diagnosis was reasonable, the RFV was described as diagnosis, if not, then as a symptom, or other statements such as "I need a refill ..." One patient concern was not counted twice as both a diagnosis and symptom, nor "I need a refill ..." and the disease.

Special situations:

Well woman/well child/check up - treated as one RFV. Patient concerns commonly associated with these visits that are rarely associated with significant underlying diseases were not counted as separate RFVs, which is consistent with CPT guidelines. For well child visits, examples included spitting up, sleep issues, eating issues, and vaccination questions. For well woman visits, examples included contraception and "Do I need a Pap smear?" Concerns that could reasonably represent an unwell person were counted separately. For well child visits, examples included diarrhea, congestion, rashes, and possible fevers. For well women visits, examples included menstrual changes, breast symptoms, or vaginal symptoms. Well man visits were generally not counted as separate RFVs.

Other classifications:

- Establish care - This was counted as a separate RFV to account for the time and cognitive work required to learn about a new patient.

- Hospital follow up - This was counted as a separate RFV to account for the time and cognitive work of learning about what happened during the hospitalization.

- "Follow up labs" - This recorded RFV was not adjusted if there was a clearly connected diagnosis in the Issues Addressed section such as anemia or hypokalemia. If the labs were connected to a chronic disease, the issue was renamed to the disease. Examples included chronic kidney disease, hyperlipidemia, and hypothyroidism.

- "Refill medications" - This was a common reason as stated by patients. This was reclassified as the acute or chronic disease the medications were presumably written if possible. For visits where "refill medications" was listed along with hypertension, migraine headaches, and gout - for example - we assumed that the medications were for the chronic diseases so we removed "refill medications" as a separate issue.

- Symptoms that are commonly connected were counted as one. Examples included runny nose/ congestion, and eye itching/eye redness.

- Social issues, healthcare system navigation issues (e.g., "my insurance is about to run out"), and concerns about family members were counted as separate RFVs.

\section{Issues Addressed (IAs)}

IAs were described as diagnosis if possible, if not, then as symptom. These were recorded as close to ICD-9 diagnoses as possible. IAs not codable with ICD-9 were classified with International Classification of Primary Care codes if possible, or codes of our own creation. Examples of the former included fear of cancer and difficult relationship with relative. Examples of the latter included falls and personal history of disease (for example, a patient with a history of colon polyps that had been removed asking about the best timing for the next colonoscopy).

Other classifications:

- Well woman/well child/check up- All treated as one IA, but not double counted. For example, common issues related to a well woman visit such as cervical cancer prevention and contraception management were counted as one IA: a well woman visit. This was based on the conventions of the CPT system. Any potentially worrisome non-"trivial" symptoms were counted as separate issues, again based on the published guidelines of the CPT system.

- Establish care and hospital follow up were not counted as a separate IA, but any discovered symptoms or diseases the physicians managed were recorded and counted.

- "Follow up labs" - If recorded, this was relabeled as the condition the labs were for or it was removed. Rarely this was not possible: for example, a visit in which the only RFV recorded was "follow up labs" and the only IA recorded was "labs normal."

- "Refill medications" or "medications reviewed" If recorded, this was relabeled as the condition 
the medications were prescribed for or it was removed.

- Treatments - The observers would occasionally record a treatment modality as an IA. Examples included "counseled on healthy lifestyle changes" or "referral to neurology." These were reclassified as the underlying disease, symptom, or concern that prompted the intervention. The intervention was recorded in the Clinical Services or Health Education/Counseling sections.

- Social/system/relative issues - These were recorded as separate IAs if the student observed the physician discussing the issue with the patient to help resolve the concern or where the treatment plan for a symptom or diagnosis was likely affected. For example, if the RFV included depression and stress at home separately, only depression may have been recorded in the IA section. If the same two RFVs were recorded, but the IA included depression and the observation that the physician reviewed counseling and shelter options for intimate partner violence, then the social issue was recorded as an IA and the counseling was recorded in the Health Education/ Counseling section as a treatment, not an IA.

- Laboratory tests -Lab tests ordered to monitor potential adverse effects of medications were captured in the lab section of our data collection instrument and not double counted as a separate IA. Point-of-care results ordered and obtained during the visit were only counted as a separate IA if a new abnormality was discovered and managed.

- Imaging - Reviewing advanced images with patients was classified as only the underlying disease detected in the image whenever possible. In a few cases, it was not clear what, if any, underlying diagnosis was suggested by the image, but the physician was given credit as a separate IA for reviewing the results with the patient.

- Paperwork - A common RFV was for paperwork to be filled out. In the IA section, paperwork was reclassified as the underlying condition necessitating the paperwork when possible. Filling out paperwork was also listed in treatment section because of the time, judgment, and expertise required in certain types of paperwork. For example, filling out paperwork for a home nebulizer was counted in the treatment section whereas filling out a form for a school sports physical with no specific diagnosis to manage was not.
- Medication adverse effects - Observed adverse effects of medications were counted as separate IAs. Ordering labs to check for a possible adverse effect were not.

- Prenatal care - Again following CPT and CMS guidelines, prenatal visits were counted as one IA if the routine issues were addressed for each visit appropriate for the gestational age. Non-"trivial" concerns were treated as separate IAs. For example, nausea occurring in the first trimester was not counted as a separate IA (also immunizations, lab discussions with no new diagnoses, genetic screening shared decision making, and Cesarean section options); hypertension in the third trimester was counted as a separate IA.

\section{Balance between RFV and IA}

The general expectation was that there would be more RFVs than IAs for each encounter. For example, if the patient reported headache, nausea, and photophobia, each was recorded by the medical student in the RFV section. Only migraine headache was recorded as an IA (if this was the final diagnosis). This data recording relationship very rarely went the other direction. An example would be a single complaint of abdominal pain where diagnoses of cholelithiasis and GERD were made.

Every IA had to have a RFV, but not the opposite, because we knew - and the observers noted that there would be times when a patient mentioned a concern that the physician did not address.

\section{Balance between IA and treatments}

Any treatment listed had to have an IA that justified it. Vaccinations were justified by well person visits or preventive services (never double counted). Brief explanations of patients' symptoms, diagnoses, medications, or tests were not counted separately in the treatment sections. More extensive education or counseling sessions were counted as a separate treatment in addition to the IA. The distinction was a judgment call made by the observers. Examples of each were given in their training sessions. For example, if the issue of smoking cessation was raised by either the physician or the patient, and the physician only wrote a prescription with little other instruction, then only the prescription was recorded. If the physician also spent a few minutes discussing strategies such as quit dates, alternative behaviors to manage stress, and social influences, this would be recorded as "Tobacco use counsel- 
ing" in the "Health Education/Counseling" section.

\section{Reportability of CPT codes}

The extended care code 99354 was applied if the observed face time was greater than 55 minutes per CMS guidelines (25 minutes for a 99214 plus at least 30 minutes toward the 99354 charge). It was assumed that the extra time allowed for all issues to be addressed, no matter the total. Therefore, all visits with 55 or more minutes of face time were counted as being fully reportable.

Coding defensible 99205/99215 codes is problematic for several reasons. Table 1 in the $E \& M$ section of the CPT guidelines states that the complexity of medical decision making includes an extensive number of diagnoses or management options, an extensive amount and/or complexity of data to be reviewed, and a high risk of complications and/or morbidity or mortality. As was stated in the body of the manuscript, there is no guidance in the body of the CPT manual that defines what an extensive number of diagnoses means. For the amount and complexity of data to be reviewed, there is also no guidance in the CPT manual of what extensive means. The CMS E\&M rules include a complicated list of data types and points that essentially mean to qualify as an extensive number of data elements, the physician must order or review labs, imaging, and either old records or other types of tests such as an electrocardiogram.

For the type of decision making, the CPT guidelines also do not explain what high complexity means explicitly in the E\&M section, but Appendix $\mathrm{C}$ of the CPT book provides some insight into the types of patients the CPT writers imagined would count as 99215 patients. There are only 2 examples of a 99205 for family medicine or internal medicine listed in Appendix C: 1) Initial office visit for a patient with disseminated lupus erythematosus with kidney disease, edema, purpura, and scarring lesions on the extremities plus cardiac symptoms, and 2) Initial outpatient encounter of a 69-year-old male with severe chronic obstructive pulmonary disease, congestive heart failure, and hypertension. Both of these patients are likely to require hospitalization. Another way to look at these examples is that they included up to 3 diseases with at least one of the diseases with a severe manifestation.

In the 99215 section, there are only 3 internal medicine/family medicine examples: 1) Office visit for an established patient with disseminated lupus ...., extensive edema of extremities, kidney disease, and weakness requiring monitored course on azathioprene and corticosteroid and complicated by acute depression, 2) Office visit with 30-year-old male, ... for 3-month history of fatigue, weight loss, intermittent fever, and presenting with diffuse adenopathy and splenomegaly, and 3) Office visit for a 70-year-old female ... with diabetes mellitus and hypertension, presenting with a 2-month history of increasing confusion, agitation, and shortterm memory loss. As in the 99205 set, each of these examples include up to roughly 3 separate issues with at least one being a severe worrisome manifestation of an existing disease or highly suspicious for a potentially fatal new diagnoses (lymphoma in example 2). The guidelines make no mention of whether the comorbidities are well controlled and therefore require no further cognitive work, or poorly controlled, therefore requiring more cognitive work to also adjust the treatment plan for those diseases.

To count every potential 99205/99215 case, every FP-patient visit that resulted in a hospitalization was counted as one of these codes. Also, every visit where potentially severe symptoms was counted as a 99205/99215. Examples included chest pain, shortness of breath, syncope, and severe weakness. Some judgment was applied by the investigators. For example, if the observer recorded "chest pain worse with inspiration" in a 20-year-old patient and there was no apparent testing or treatment for this symptom, other than a common medication such as an anti-inflammatory, than this visit was not counted as a 99205/ 99215. For visits that included a worrisome symptom, if there were more than 3 issues addressed beyond the severe symptom or exacerbated chronic disease, this visit was counted as including more cognitive work than a 99205/ 99215 allows to be reported. This approach is consistent with the description of the degree of risk explained in the CMS E\&M guidelines. 


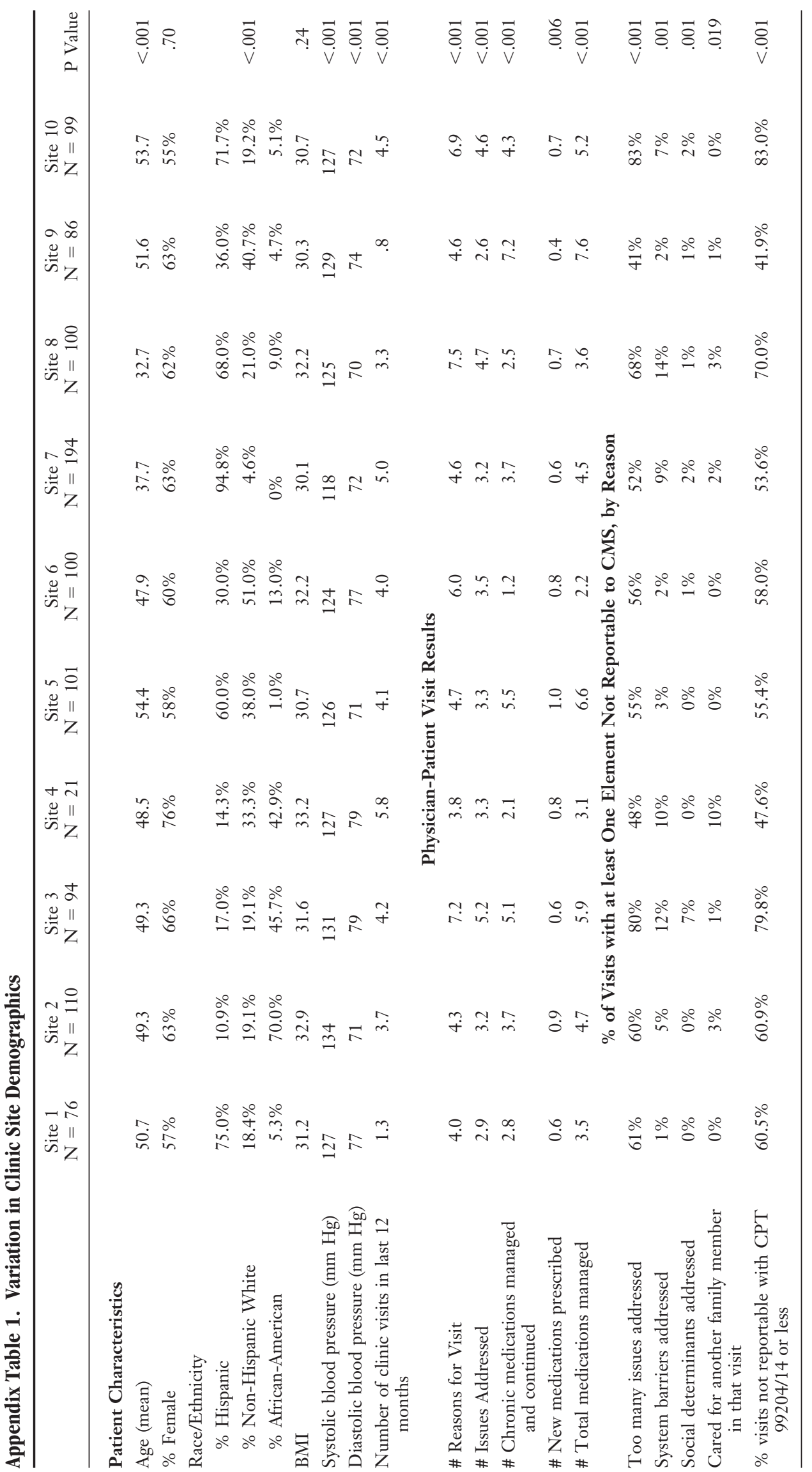

